\title{
Quantitative estimates of relationships between geomagnetic activity and equatorial spread-F as determined by TID occurrence levels
}

\author{
G. G. Bowman and I. K. Mortimer
}

Department of Physics, The University of Queensland Brisbane, Qld., 4072, Australia

(Received June 11, 1999; Revised December 22, 1999; Accepted May 29, 2000)

\begin{abstract}
Using a world-wide set of stations for 15 years, quantitative estimates of changes to equatorial spread-F (ESF) occurrence rates obtained from ionogram scalings, have been determined for a range of geomagnetic activity (GA) levels, as well as for four different levels of solar activity. Average occurrence rates were used as a reference. The percentage changes vary significantly depending on these subdivisions. For example for very high GA the inverse association is recorded by a change of $-33 \%$ for $R_{z} \geq 150$, and $-10 \%$ for $R_{z}<50$. Using data for 9 years for the equatorial station, Huancayo, these measurements of ESF, which indicate the presence of TIDs, have also been investigated by somewhat similar analyses. Additional parameters were used which involved the local times of GA, with the ESF being examined separately for occurrence pre-midnight (PM) and after-midnight (AM). Again the negative changes were most pronounced for high GA in $R_{z}$-max years $(-21 \%)$. This result is for PM ESF for GA at a local time of 1700 . There were increased ESF levels $(+31 \%)$ for AM ESF in $R_{z}$-min years for high GA around 2300 LT. This additional knowledge of the influence of GA on ESF occurrence involving not only percentage changes, but these values for a range of parameter levels, may be useful if ever short-term forecasts are needed. There is some discussion on comparisons which can be made between ESF results obtained by coherent scatter from incoherent-scatter equipment and those obtained by ionosondes.
\end{abstract}

\section{Introduction}

Periodic tilted isoionic contours, recorded at the times of equatorial spread-F (ESF) occurrence, were detected by an early investigation (Rottger, 1973). It is now generally accepted that travelling ionospheric disturbances (TIDs) have a role to play in the occurrence of ESF. Also, Singh et al. (1997a) report that there is growing evidence that the Rayleigh Taylor Instability (RTI) is seeded by the atmospheric gravity waves (AGWs) which are responsible for the TIDs (see also Kelley et al., 1981; Kelley, 1989; Hysell et al., 1990; Kelley and Hysell, 1991; Singh et al., 1997b). It now seems likely, as is the case in mid latitudes (Bowman, 1990 and 1991a), that most of the spread-F traces on equatorial ionograms are recorded because of the existence of periodic tilted isoionic surfaces generated by the passage of TIDs. The evidence for this is relatively recent, these spread traces being identified as those expected from radiowave specular reflections from significantly-large off-vertical angles. For example Flaherty et al. (1996) state "Large spreads in angle of arrival, as much as $20 \mathrm{deg}$, were also seen. The strength of the off great circle returns are comparable to the strength of signals arriving from the direction of the great circle and are believed to result from total reflections from horizontal gradients in the ionosphere." Wright et al. (1996) mention that "The total-reflection interpretation is shown to be consistent with rocket and satellite data" (see also Argo and Kelley, 1986; Sales et al., 1996; Weber et al., 1996). In

Copy right(C) The Society of Geomagnetism and Earth, Planetary and Space Sciences (SGEPSS); The Seismological Society of Japan; The Volcanological Society of Japan; The Geodetic Society of Japan; The Japanese Society for Planetary Sciences. addition subtle phase-path measurements for equatorial regions show the TID wave amplitudes are larger when ESF is present (Subbarao and Krishna Murthy, 1994) which indicates that lower-amplitude TIDs are present at other times, as has been found for mid latitudes (Bowman, 1995a). Thus ESF is only recorded on ionograms when TID wave amplitudes are sufficiently large. In view of the experimental results it is probably reasonable to assume that equatorial ionograms give evidence mainly of the existence of TIDs with amplitudes appropriate for radiowaves to be reflected from relatively-large off-vertical angles. In contrast the coherent scatter associated with incoherent-scatter equipment gives information on the small-scale irregularities (SSIs) and not on the more-ordered periodic wavelike structures produced by the TIDs (Rottger, 1973). Ionograms and incoherent-scatter equipment record information on quite different aspects of the same ionosphere. Spread-F was originally defined in terms of these extra ionogram traces, although the term is now more generally used to apply to nighttime disturbance conditions. Since TIDs are now recognised as seeding the RTI and may possibly always be needed for this instability to operate, any investigation into the occurrence of equatorial TIDs would seem to be important for the study of ESF. In any case, whether or not they are always needed, a study of equatorial TIDs in their own right, would seem appropriate. However the characteristics of these two equatorial parameters (TIDs and SSIs) may not always be identical so that in this paper the term ESF will be modified and sometimes where appropriate called $\mathrm{ESF}_{\mathrm{T}}$ for ionogram TID events and $\mathrm{ESF}_{\mathrm{S}}$ for coherent-scatter events. A section will be devoted to 
EQUATORIAL SPREAD-F VERSUS GEOMAGNETIC ACTIVITY

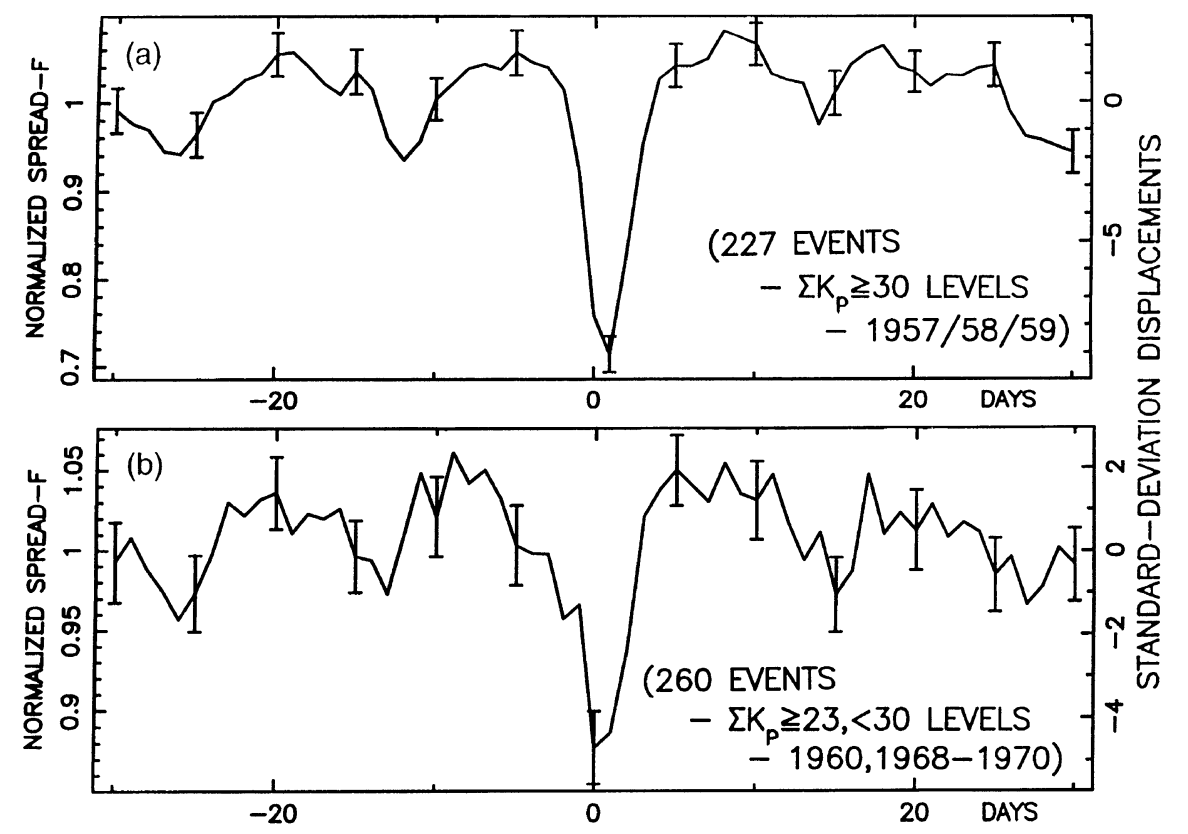

Fig. 1. Equatorial ESF activity relative to (a) $\sum K_{P} \geq 30$ for $R_{z}>150$, and (b) $\sum K_{P} \geq 23,<30$ for $R_{z} \geq 100,<150$.

Table 1. Equatorial stations.

\begin{tabular}{|c|c|c|c|}
\hline & \multicolumn{2}{|c|}{ Geomagnetic coordinates } & \multirow[b]{2}{*}{ Dip } \\
\hline & Latitude & Longitude & \\
\hline Huancayo & -0.6 & 353.8 & 1.4 \\
\hline Trivandrum & -1.1 & 146.4 & -1.6 \\
\hline Kodaikanal & 0.6 & 147.1 & 2.5 \\
\hline Tiruchirapalli & 1.0 & 148.3 & 3.8 \\
\hline La Paz & -5.0 & 00.9 & -5.2 \\
\hline Djibouti & 7.1 & 113.5 & 6.1 \\
\hline Ibadan & 10.6 & 74.6 & -7.0 \\
\hline Madras & 3.2 & 150.1 & 9.3 \\
\hline Bangkok & 2.4 & 169.9 & 10.4 \\
\hline Manila & 3.4 & 189.8 & 14.5 \\
\hline Baguio & 5.0 & 88.6 & -14.7 \\
\hline Dakar & 21.6 & 54.6 & 16.9 \\
\hline Singapore & -10.1 & 172.7 & -18.3 \\
\hline Hyderabad & 7.5 & 148.8 & 19.4 \\
\hline
\end{tabular}

examining any differences between these parameters. These two systems have been compared by Argo and Kelley (1986). They find $\mathrm{ESF}_{\mathrm{S}}$ recorded by coherent-scatter to be detected as the ionospheric layer is rising whereas the $\mathrm{ESF}_{\mathrm{T}}$ is recorded on ionograms as the layer is falling. More recently Sastri (1999) finds that ionogram-recorded spread-F $\left(\mathrm{ESF}_{\mathrm{T}}\right)$, which commences around midnight does not appear to be related to significant post-sunset height rises which occur earlier. This paper reports on investigations into possible influences geomagnetic activity (GA) might have on $\mathrm{ESF}_{\mathrm{T}}$.

It has been shown that a well-defined relationship exists between $\mathrm{ESF}_{\mathrm{T}}$ and enhanced GA, particularly at certain local times (LT) for the station being considered. For Huancayo those times are 1100 and 1800 . The relationship is inverse except for $\mathrm{ESF}_{\mathrm{T}}$ in the pre-sunrise hours especially in sunspotminimum years (Bowman, 1995b). Another aspect which emphasises the inverse relationship is that sometimes a high level of $\mathrm{ESF}_{\mathrm{T}}$ on one night is changed to zero $\mathrm{ESF}_{\mathrm{T}}$ activity on the following night. On these occasions there is a significant increase in geomagnetic activity in the daylight hours preceding this night of no $\mathrm{ESF}_{\mathrm{T}}$ occurrence (see figures 2, 3, 4 of Bowman, 1998). Although this $\mathrm{ESF}_{\mathrm{T}} / \mathrm{GA}$ relationship is recorded at a high level of significance, it is not known, for example, to what extent $\mathrm{ESF}_{\mathrm{T}}$ levels change for different GA levels. This present investigation is an attempt to increase knowledge of this relationship with analyses which allow estimates of percentage changes after various levels of GA. Account is also taken of the local time of occurrence of GA, as well as sunspot number $\left(R_{z}\right)$ levels. In addition some analyses consider pre-midnight (PM) ESF occurrence separately from after-midnight (AM) occurrences. Since $\mathrm{ESF}_{\mathrm{T}}$ occurrence rates change significantly annually and during the solar cycle (Bowman, 1993a) it has been necessary to normalise the data. This was done by dividing events in each 60 day interval by the average value for that interval.

The $\mathrm{ESF}_{\mathrm{T}}$ occurrence was determined from the published hourly values of $f_{0} F_{2}$. Whether or not the descriptive letter was $\mathrm{F}$ or $\mathrm{Q}$ the spread-F events indicated were used. Bowman (1991b) has shown (at least for mid latitudes) that frequency spread and range spread are different aspects of the same phenomenon. Also, the events indicated by these symbols were used irrespective of whether or not a definite value was available for $f_{0} F_{2}$. Two different sets of analyses will be reported here. The first combines data from equatorial stations 


\section{EQUATORIAL SPREAD-F VERSUS GEOMAGNETIC ACTIVITY}
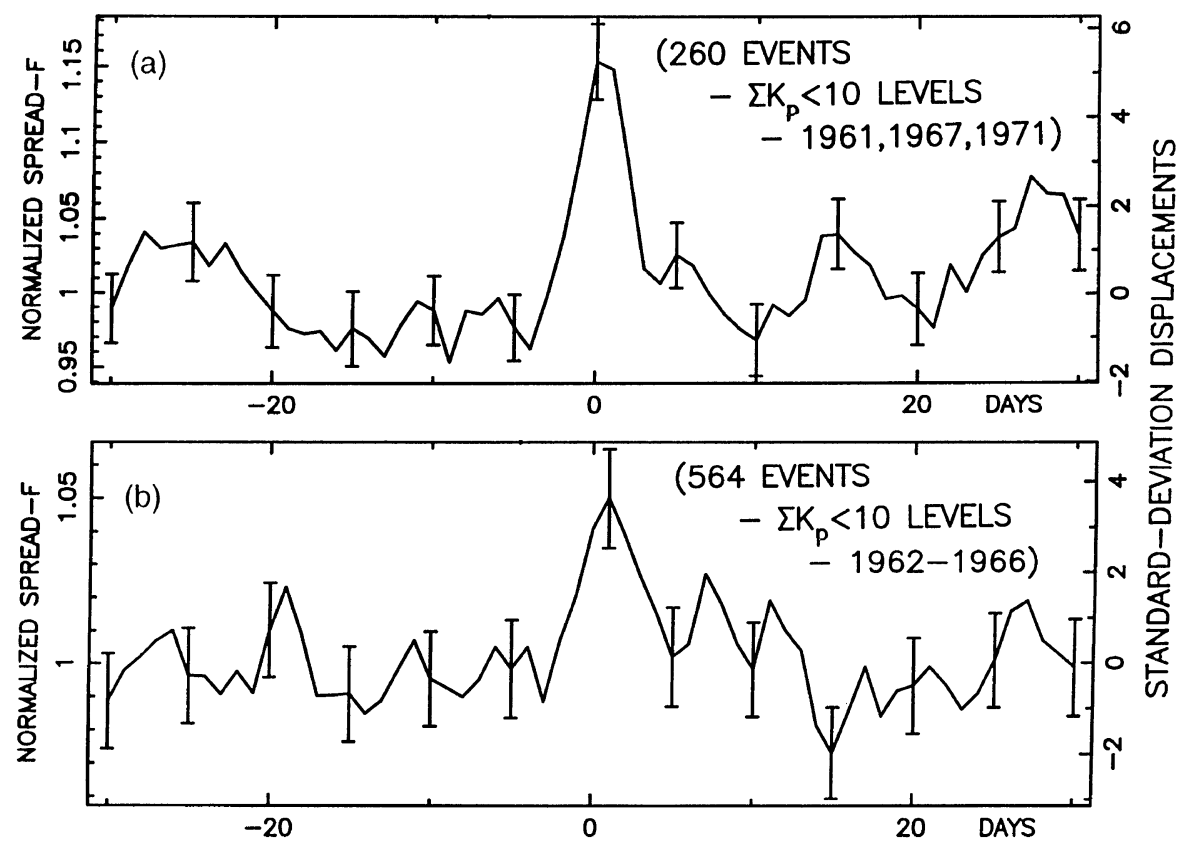

Fig. 2. Equatorial ESF activity relative to (a) $\sum K_{P}<10$ for $R_{z} \geq 50,<100$ and (b) $\sum K_{P}<10$ for $R_{z}<50$.

Table 2. Equatorial spread-F range of stations.

\begin{tabular}{lrrrr}
\hline$\sum K_{P}$ Levels & \multicolumn{4}{c}{ Percentage change } \\
\cline { 2 - 5 } & $R_{z} \geq 150$ & $\begin{array}{r}R_{z} \geq 100 \\
<150\end{array}$ & $\begin{array}{r}R_{z} \geq 50 \\
<100\end{array}$ & $R_{z}<50$ \\
\hline Very high & $-33(227)$ & $-20(168)$ & $-21(105)$ & $-10(123)$ \\
High & $-27(169)$ & $-18(122)$ & $-24(88)$ & $-12(109)$ \\
Medium & $-7(257)$ & $-13(260)$ & $-12(137)$ & $-6(240)$ \\
Low & $+30(94)$ & $+18(254)$ & $+15(260)$ & $+5(564)$ \\
\hline
\end{tabular}

around the world, to obtain quantitative estimates of $\mathrm{ESF}_{\mathrm{T}}$ changes, while the other investigation allows the calculation of similar estimates for a single station (Huancayo). Further details, including information on additional subdivisions of the parameters used will be given later.

\section{Methods of Analysis}

Superposed-epoch (SE) methods have been used throughout. Data representing the daily occurrence of either worldwide ESF or Huancayo ESF, as recorded by ionograms, were examined relative to controls representative of GA levels. Different $R_{z}$ levels were also considered for these controls. The investigations embraced the centre day (CD) \pm 100 days (i.e. 201 days), but in the examples to be given here only the central sections of the final plots ( $\mathrm{CD} \pm 30$ days) will be shown. The right-hand label on the plot of each analysis was obtained by calculating the standard-deviation of the SE summations (201 values), thus allowing a scale which indicates the standard-deviation displacements from the mean of all the summations. The percentage changes of ESF levels associated with GA were available on the left-hand labels by considering levels relative to the mean value which is indicated by the zero standard-deviation displacements. Standard-error bars, which were also available, are shown in the figures to be presented.

\section{Results}

The percentage changes from all the plots produced will be given in tabular form. However sample plots will also be presented.

\subsection{ESF for a group of equatorial stations}

The analyses have used ionogram data from all available equatorial stations which have dip angles $\leq 20^{\circ}$. The number of stations in any interval varied depending on the availability of data. As many as 14 stations were used with the average number being 10 . The geomagnetic coordinates of these stations are listed in Table 1. The period was 1957 to 1971 subdivided into different levels of $R_{z}\left(1957,58,59\right.$ for $R_{z} \geq 150$; $1960,1968,69,70$ for $100 \leq R_{z}<150 ; 1961,1967,1971$ for $50 \leq R_{z}<100$; and 1962-1966 for $R_{z}<50$ ). $K_{P}$ sum levels were used for GA, with values $\geq 30$ classified as very high; $\geq 30<40$ as high; $\geq 23<30$ as medium and $<10$ as low. A summary of the percentage changes (with event numbers in brackets) is presented in Table 2 . The association is 


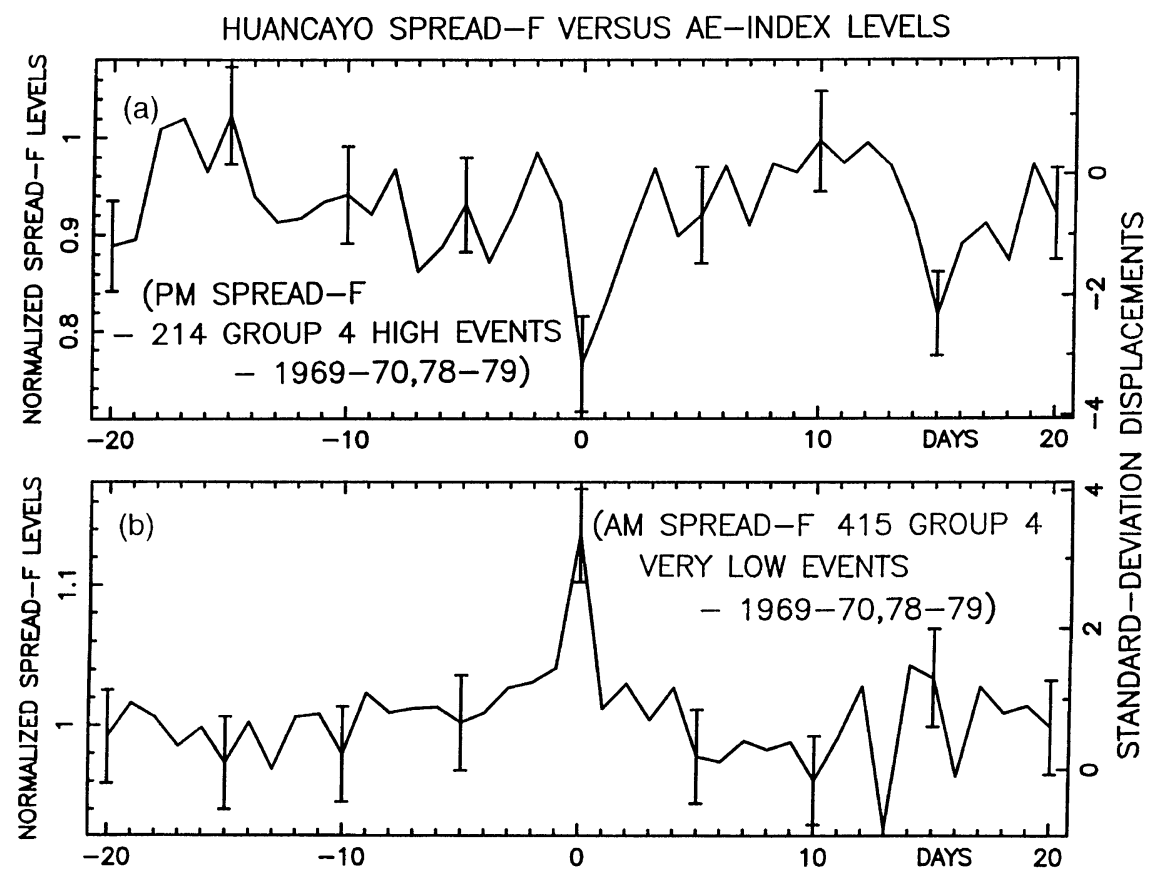

Fig. 3. Huancayo PM ESF activity relative to (a) high AE-index levels and (b) very low events, both near 1700 LT in $R_{z}$-max years.

Table 3. Time ranges for AE indices.

\begin{tabular}{lcccc}
\hline & \multicolumn{4}{c}{ Group numbers } \\
\hline Times & 1 & 2 & 3 & 4 \\
UT & $03-05$ & $09-11$ & $15-17$ & $21-23$ \\
Centre (LT) & 2300 & 0500 & 1100 & 1700 \\
Huancayo & & & & \\
\hline
\end{tabular}

most pronounced in sunspot maximum $\left(R_{z}\right.$-max) years, the changes being quite significant (around 30 percent) for both the inverse (high $\sum K_{P}$ ) and direct (low $\sum K_{P}$ relationships. The estimates for sunspot-minimum $\left(R_{z}\right.$-min) years are varied but are generally around half those for $R_{z}$-max years. For different $R_{z}$ levels Figures 1(a) and 1(b) illustrate sample plots for the inverse relationship for enhanced GA, while Figures 2(a) and 2(b) show that the effect is reversed to a direct relationship for very low GA. Some indication of the importance of this relationship can be gained by considering the fact that the polarity of the displacements reverse from negative to positive at around $\sum K_{P}=20$. This probably means that average levels of ESF have been influenced by this level of GA. The right-hand scales in the figures and the error bars attest to the high statistical significance of the results as was found earlier (Bowman, 1995b). For example Figure 1(a) shows a standard-deviation displacement of $9 \sigma$, while Figure 2(a) gives a value of $5 \sigma$. However, these qualitative results are not suitable for accurate short-term forecasts. The left-hand scale allows a quantitative estimate of the magnitude of the influence of the GA. The extraction of the data from these equatorial stations was commenced some time ago and daily occurrence rates were taken from midnight to midnight, thus embracing the after-midnight (AM) period of one night and the pre-midnight (PM) period of the next night.
As illustrated by Figs. 1 and 2 the analyses for this subsection give two minima or maxima, because of the way the daily values of ESF represent sections of two nights. It is now realised that it is better to group the PM and AM periods of the same night together.

For these worldwide equatorial stations since $\mathrm{ESF}_{\mathrm{T}}$ occurs at night it has been necessary to determine occurrence rates in $\mathrm{LT}$, whereas the $\sum K_{P}$ values refer to universal time (UT). The global distribution of these stations involves locations to the east and west of the UT reference longitude. Consequently the station time zones will be located mainly in the UT zone, but with some overlap of a few hours when these stations are considered collectively. However the time resolution for the analysis is in days. Despite these different time zones, results at significant levels are found. Thus these can be used for forecast purposes for $\mathrm{ESF}_{\mathrm{T}}$ when considered on a global scale.

\subsection{ESF for Huancayo}

For analyses involving the single station (Huancayo: $-00.57,353.84$ geomagnetic coordinates) GA was measured by the AE index. The GA was classified as high when the $\mathrm{AE}$ index was $>500 \mathrm{nT}$; medium if $>200 \geq 500 \mathrm{nT}$; low if $>100 \leq 200 \mathrm{nT}$ and very low if $\leq 100 \mathrm{nT}$. Additional controls were obtained by subdivisions involving local times (LT) of the station plus a distinction made between PM and AM intervals; for the analyses the AM period was given the date of the PM period which preceded it. Table 3 lists four groups of three consecutive hours in UT representing 2 hour intervals. The centre hours of these groups are also listed in Table 3 in LT. The highest values for each of the three indices associated with these groups were used as controls. Group 4 interval was arranged to be in the same day (UT) for the convenience of the analyses. Consequently the centre time (LT) is at 1700 rather than 1800 . In view of these additional variables only two $R_{z}$ levels were used $\left(R_{z}>75 ; R_{z} \max \right.$ 
HUANCAYO SPREAD-F VERSUS AE-INDEX LEVELS
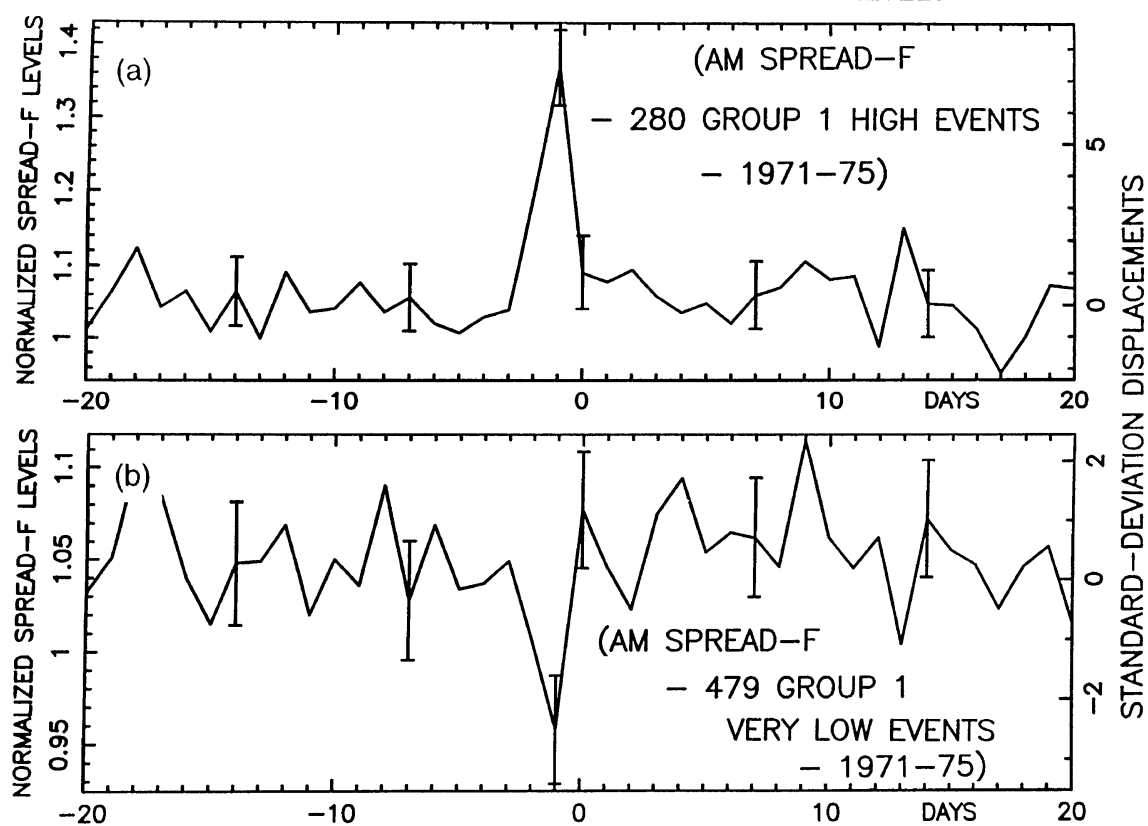

Fig. 4. Huancayo AM ESF activity relative to (a) high AE-index levels and (b) very low events, both near 2300 LT in $R_{z}$-min years.

Table 4. Huancayo PM spread-F $R_{z}$-maximum years.

\begin{tabular}{lrrrr}
\hline $\begin{array}{l}\text { AE-index } \\
\text { level }\end{array}$ & \multicolumn{4}{c}{ Percentage change } \\
\cline { 2 - 5 } & Group 1 & Group 2 & Group 3 & \multicolumn{1}{c}{ Group 4 } \\
\hline High & $-9(181)$ & $-7(223)$ & $-18(272)$ & $-21(214)$ \\
Medium & $-6(440)$ & $-5(493)$ & $-7(444)$ & $-9(466)$ \\
Low & $0(282)$ & $-3(266)$ & $+5(292)$ & $0(253)$ \\
Very low & $+5(447)$ & $+11(369)$ & $+13(330)$ & $+20(415)$ \\
\hline
\end{tabular}

Table 5. Huancayo AM spread-F $R_{z}$-maximum years.

\begin{tabular}{lcccr}
\hline $\begin{array}{l}\text { AE-index } \\
\text { level }\end{array}$ & \multicolumn{4}{c}{ Percentage change } \\
\cline { 2 - 5 } & Group 1 & Group 2 & Group 3 & Group 4 \\
\hline High & $+10(181)$ & $-8(223)$ & $-9(272)$ & $-18(214)$ \\
Medium & $-7(440)$ & $-3(493)$ & $-11(444)$ & $-8(466)$ \\
Low & $-4(282)$ & $+1(266)$ & $+1(292)$ & $0(253)$ \\
Very low & $-2(447)$ & $+6(369)$ & $+11(330)$ & $+17(415)$ \\
\hline
\end{tabular}

and $R_{z} \leq 75 ; R_{z} \min$ ) so as to reduce the number of possible combinations. The $R_{z}$ max years used were 1969,1970 and 1978,1979 while the years for $R_{z}$ min were $1971-1975$. No AE index values were available for 1976 and 1977.

The details of all the results are listed in Tables 4 and 5 for $R_{z}$-max years and Tables 6 and 7 for $R_{z}$-min years. These quantitative estimates reflect generally the qualitative results found earlier (Bowman, 1995b). However quite apart from the quantitative values available, information is also detailed
Table 6. Huancayo PM spread-F $R_{z}$-minimum years.

\begin{tabular}{lrrrr}
\hline $\begin{array}{l}\text { AE-index } \\
\text { level }\end{array}$ & \multicolumn{4}{c}{ Percentage change } \\
\cline { 2 - 5 } & Group 1 & Group 2 & Group 3 & Group 4 \\
\hline High & $-4(280)$ & $-1(363)$ & $-12(387)$ & $-12(341)$ \\
Medium & $-2(571)$ & $-3(570)$ & $-1(575)$ & $-3(590)$ \\
Low & $0(361)$ & $-1(349)$ & $0(338)$ & $+8(340)$ \\
Very low & $+6(479)$ & $+11(392)$ & $+14(364)$ & $+13(418)$ \\
\hline
\end{tabular}

Table 7. Huancayo AM spread-F $R_{z}$-minimum years.

\begin{tabular}{lrrrr}
\hline $\begin{array}{l}\text { AE-index } \\
\text { level }\end{array}$ & \multicolumn{5}{c}{ Percentage change } \\
\cline { 2 - 5 } & Group 1 & Group 2 & Group 3 & Group 4 \\
\hline High & $+31(280)$ & $+12(363)$ & $+7(387)$ & $+4(341)$ \\
Medium & $0(571)$ & $-2(570)$ & $+1(575)$ & $-1(575)$ \\
Low & $-12(361)$ & $-9(349)$ & $0(338)$ & $-4(340)$ \\
Very low & $-9(479)$ & $-4(392)$ & $-5(364)$ & $+1(418)$ \\
\hline
\end{tabular}

on the influence of different levels of GA. In addition there is more detail on the response of the nighttime equatorial ionosphere to GA which occurs at different times of the day. Except for AM spread-F in $R_{z}$-min years (Table 7 ) and to a lesser extent in $R_{z}$-max years (Table 5 ), the influence is more pronounced for groups 3 and 4 which are representative of the local times of 1100 and 1700 respectively (Table 3). For example for PM spread for group 4 in $R_{z}$-max years (Table 4 ) there is a negative percentage change of -21 for high GA 
AE INDEX VERSUS BRISBANE AM SPREAD-F EVENTS 1971-1975

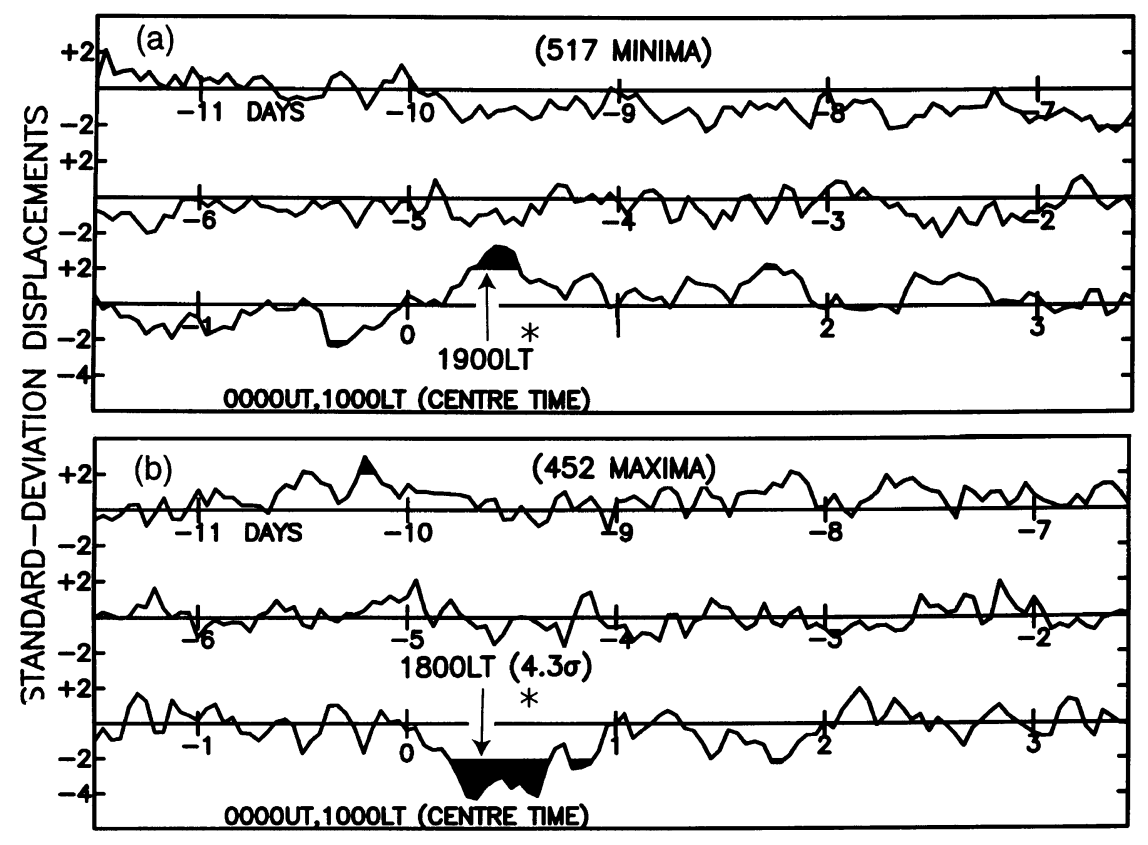

Fig. 5. Short-term relationships between Brisbane spread-F occurrence and GA for (a) spread-F minima and (b) spread-F maxima.

with a positive value of +20 when the activity is very low. The plots for these results are shown in Figs. 3(a) and 3(b) respectively. For the AM spread in $R_{z}$-min years (Table 7) the positive relationships which occur for high GA are shown by contrast to be dominant at local times of 2300 and 0500 (Table 3), with positive percentage changes of +31 and +12 respectively. Earlier Bowman (1995b) found the maximum influence for Huancayo occurred at local times of 2200 and 0800. For both investigations the positive displacements are more prominent for high GA an hour or two before midnight. The group 1 results for high and very low GA in $R_{z}$-min years are shown in Figs. 4(a) and 4(b) respectively (Table 7). In $R_{z}$ max years a positive percentage change of +10 is noted for high GA around the local time of 2300 (group 1 of Table 5).

Suggested mechanisms which might account for these relationships, which were proposed earlier (Bowman, 1995b and 1998), will be mentioned briefly in the Discussion and Conclusions section.

\section{A Comparison with Coherent-Scatter Results}

It would be interesting to ascertain to what extent the coherent-scatter results for SSIs (Scherliess and Fejer, 1997; Fejer and Scherliess, 1997; Fejer et al., 1999) differ from the TID results particularly related to the modification of occurrence levels produced by GA. Existing literature allows some comments to be made on this comparison. The influence of the vertical plasma drift (Fejer et al., 1999) on TID occurrence is not known, although it has been suggested that wave amplitudes become larger at higher $\mathrm{F}_{2}$-layer heights because of neutral-density changes (Bowman, 1993a). The annual and sunspot-cycle variations of ESF before and after midnight seem to be in agreement for these two parameters (SSIs and TIDs) (Bowman, 1993a; Fejer et al., 1999). Concerning associations with GA, Fejer et al. (1999) report on literature which indicates that $\mathrm{ESF}_{\mathrm{S}}$ is suppressed following
GA around midday but is enhanced when it occurs in the late afternoon hours. For $\mathrm{ESF}_{\mathrm{T}}$ experimental evidence reported by Bowman (1995b, 1998), and also in this present paper, indicate at a high level of significance a suppression of $\mathrm{ESF}_{\mathrm{T}}$ for GA not only around 1200 LT but also around 1800 LT, this last observation being at variance with the $\mathrm{ESF}_{\mathrm{S}}$ results. For example, the probability that the $6.1 \sigma$ value at $1800 \mathrm{LT}$ on Fig. 4 of Bowman (1998) is a random displacement is $0.0^{6} 1$ percent (Croxton, 1949), the final array in the analysis involved being shown to have a normal distribution.

What seems to be another important aspect is the fact that papers on $\mathrm{ESF}_{\mathrm{S}}$ generally assume that the phenomenon is unique to equatorial regions, but some characteristics associated with $\mathrm{ESF}_{\mathrm{T}}$ are found in mid latitudes (see tables 2 and 3 of Bowman, 1998). Figure 5 is presented here to support the fact that the suppression of $\mathrm{ESF}_{\mathrm{T}}$ for $\mathrm{GA}$ near 1800 LT can also be observed for mid-latitude ionogramrecorded spread-F. At Brisbane (-35.7, 226.9 geomagnetic) for 1971-1975 and when spread-F occurrence after-midnight (AM) was $\leq 0.5$ times the average occurrence every 2 months (i.e. when spread-F is suppressed) a SE analyses (explained in detail earlier, Bowman, 1993b, 1994) indicates significant enhanced GA occurrence in the late-afternoon and earlyevening hours (Fig. 5(a)). Figure 5(b) gives the negative displacements for spread-F maxima for levels $\geq 1.33$ times the average values. On this figure the probability that the $4.3 \sigma$ value at $1800 \mathrm{LT}$ is a random displacement is 0.002 percent (Croxton, 1949) with again the final SE array (involving centre time \pm 30 days) having a normal distribution. The asterisks on Fig. 5 indicate the location of local midnight. Even daytime disturbances at Brisbane, which involve TIDs, have been shown (Bowman, 1994) to be related inversely to GA a few hours earlier. 


\section{Discussion and Conclusions}

For groups of equatorial stations distributed around the world, Table 2 shows for $R_{z} \geq 150$ a percentage change of -33 for very high GA, -27 when it is high, -7 for medium $\left(23 \leq \sum K_{P}<30\right)$, and finally +20 for low. For the singlestation analyses (Huancayo) and $R_{z}$-max years Table 4 gives a percentage change of -21 for high GA, -9 when the level is medium, zero for low, and +20 for very low. The sign reversal of the association from negative to positive, which occurs for low AE-index values (between 100 and $200 \mathrm{nT}$ ) is comparable with a 3-hourly $K_{P}$ index of 2.5 (or 20 if $\sum K_{P}$ for 8 intervals is considered) (Rostoker, 1991). In Subsection 3.1, 20 was indicated as the possible crossover $\sum K_{P}$ value for the analyses involving the group of stations. Thus Tables 2 and 4 give what appears to be a consistent crossover level (from negative to positive) for GA. It seems important to try to understand why this reversal is recorded by the analyses used. One interpretation which seems unlikely is that the ESF occurrence rate is increased significantly for very low GA. However it is suggested here, since there is evidence that the influence decreases with lower levels of GA, that GA has an influence which is so important that the average ESF levels are also influenced, the GA level being around $\sum K_{P}=20$. This would mean that when comparisons are made with these average levels the influence will appear to be positive for $\sum K_{P}<20$, and particularly for very low GA levels such as AE-index levels $\leq 100 \mathrm{nT}$.

The analyses for this paper have allowed quantitative estimates of percentage changes to $\mathrm{ESF}_{\mathrm{T}}$ taking into consideration levels of sunspot activity, GA and local times of GA. One analysis considered a group of equatorial stations (Table 1) distributed around the world while another concentrated on a single station (Huancayo). Despite the existence of some longitudinal differences for the group, such as the slightly different annual variations (Bowman, 1993a) the $\mathrm{ESF}_{\mathrm{T}}$ for the group had a well-defined relationship with GA (Fig. 1). In any case responses to GA from the widely spaced stations of Huancayo and Manila have been shown to be essentially the same (Bowman, 1995b). The suggested mechanisms for both the negative and positive responses involving neutral-particle densities and large scale travelling ionospheric disturbances (LS-TIDs) respectively have been dealt with in some detail in earlier papers (Bowman, 1995b and 1998) and so will not be discussed further here. However it seems worth noting that recent results (Deminova et al., 1998; Hajkowicz, 1999) have found that height rises and electron-density depletions associated with LS-TIDs can be tracked from auroral-zone regions all the way to the equator. Thus they may be responsible for at least some of the pre-sunrise ESF. Irrespective of what mechanisms are involved in these changes, the quantitative estimates derived here are useful if short-term forecasts are ever needed of nighttime equatorial disturbance conditions involving TIDs.

The presentation has been careful to distinguish the associations reported here representing changes associated with ionosonde results of equatorial TID occurrence levels from the results relating to SSIs obtained from coherent scatter using incoherent-scatter equipment. It is suggested that results which become available from the recent coherent scatter investigations (Scherliess and Fejer, 1997; Fejer and
Scherliess, 1997) be compared with at least the qualitative $\mathrm{ESF}_{\mathrm{T}}$ results. For example, for the coherent- scatter results, at what level of $\sum K_{P}$ does the relationship change from negative to positive? These may not necessarily be identical particularly as ionosonde results similar to those for equatorial regions are also found for mid-latitude stations (see Section 4 and Fig. 5).

It is accepted by most investigators that in equatorial regions the RTI has a central role in the generation of SSIs, as detected by coherent scatter. However it seems reasonable to question the view found in some papers that the spread traces found on equatorial ionograms are also associated with the RTI. As detailed in the Introduction the experimental evidence now indicates that these traces result primarily from radiowave off-vertical specular reflections from TID structures. This allows the equatorial TIDs to be considered separately. Rastogi (1986) has shown that VHF irregularities are sometimes present when no spread is recorded on equatorial ionograms. As earlier papers have shown (Bowman, 1993a, 1995b, 1998) similarities exist for TID occurrence characteristics in both equatorial and mid-latitude locations (see also Fig. 5 of this paper). It therefore does not seem unreasonable to consider the possibility that similar mechanisms may operate for certain aspects of spread-F occurrence in these two latitude regions. For example the annual and sunspot-cycle variations for AM equatorial spread-F and mid-latitude spread-F are essentially the same (Bowman, 1960 and 1993a).

Acknowledgments. The authors would like to thank D. Dearden for valuable assistance with the data analyses.

\section{References}

Argo, P. E., M. C. Kelley, Digital ionosonde observations during equatorial spread-F, J. Geophys. Res., 91, 5539-5555, 1986.

Bowman, G. G., Further studies of "spread-F” at Brisbane-I., experimental, Planet. Space Sci., 2, 133-149, 1960.

Bowman, G. G., A review of some recent work on mid-latitude spread-F occurrence as detected by ionosondes, J. Geomag. Geoelectr., 42, 109138, 1990.

Bowman, G. G., Nighttime mid-latitude travelling ionospheric disturbances associated with mild spread-F conditions, J. Geomag. Geoelectr., 43, 899-920, 1991a.

Bowman, G. G., Ionospheric frequency spread and its relationship with range spread in mid-latitude regions, J. Geophys. Res., 96, 9745-9753, $1991 b$.

Bowman, G. G., The influence of the upper-atmosphere neutral particle density on the occurrence of equatorial spread-F, Ann. Geophys., 11 624-633, 1993a.

Bowman, G. G., Daytime and nighttime mid-latitude ionospheric disturbances and their delayed occurrence after geomagnetic activity, Indian J. Radio Space Phys., 22, 1-10, 1993b.

Bowman, G. G., Short-term delays in the occurrence of mid-latitude ionospheric disturbances following other geophysical and solar events, J. Ge omag. Geoelectr., 46, 297-309, 1994.

Bowman, G. G., Multiplicity of travelling disturbances in the nighttime midlatitude $F_{2}$-region ionosphere, Indian J. Radio Space Phys., 24, 91-96, 1995 a.

Bowman, G. G., Short-term delays in ionogram-recorded equatorial spreadF occurrence after both solar and geomagnetic activity, Ann. Geophys. 13, 1331-1342, 1995 b.

Bowman, G. G., Short term delays (hours) of ionospheric spread-F occurrence, at a range of latitudes, following geomagnetic activity, J. Geophys. Res., 103, 11,627-11,634, 1998.

Croxton, F. E., Tables of Areas in Two Tails and One Tail of the Normal Curve, Prentice-Hall, Inc., London, 1949.

Deminova, G. F., V. M. Shashunkina, and E. E. Goncharova, A global 
empirical model of effects of large-scale internal gravity waves in the night-time ionosphere, J. Atmos. Solar-Terr. Phys., 60, 227-245, 1998.

Fejer, B. G., L. Scherliess, Empirical models of storm time equatorial zonal electric fields, J. Geophys. Res., 102, 24,047-24,056, 1997.

Fejer, B. G., L. Scherliess, and E. R. de Paula, Effects of the vertical plasma drift velocity on the generation and evolution of equatorial spread F, $J$. Geophys. Res., 104, 19,859-19,869, 1999.

Flaherty, J. P., M. C. Kelley, C. E. Seyler, and T. J. Fitzgerald, Simultaneous VHF and transequatorial HF observations in the presence of bottomside equatorial spread F, J. Geophys. Res., 101, 26,811-26,818, 1996.

Hajkowicz, L. A., Monitoring ionospheric response to auroral electrojet activity from sub-auroral to equatorial latitudes in the east Asian-Australian longitudinal sector over a solar cycle (1978-1986), J. Atmos. Solar-Terr. Phys., 61, 857-866, 1999.

Hysell, D. L., M. C. Kelley, W. E. Swartz, and R. F. Woodman, Seeding and layering of equatorial spread $\mathrm{F}$ by gravity waves, J. Geophys. Res., 95, $17,253-17,260,1990$.

Kelley, M. C., The Earth's Ionosphere, Academic, San Diego, Calif., 1989.

Kelley, M. C. and D. L. Hysell, Equatorial spread-F and neutral atmospheric turbulence: a review and a comparative anatomy, J. Atmos. Terr. Phys., 53, 695-708, 1991.

Kelley, M. C., M. F. Larson, and C. LaHoz, Gravity wave initiation of equatorial spread F; A cade study, J. Geophys. Res., 86, 9087-9100, 1981.

Rastogi, R. G., On the occurrence of equatorial spread-F in the evening hours, J. Atmos. Terr. Phys., 48, 687-693, 1986.

Rostoker, G., A quantitative relationship between $\mathrm{AE}$ and $K_{P}, J$. Geophys.
Res., 96, 5853-5857, 1991.

Rottger, J., Wavelike structures of large scale equatorial spread F irregularities, J. Atmos. Terr. Phys., 35, 1195-1206, 1973.

Sales, G. S., B. W. Reinisch, J. L. Scali, and C. Dozois, Spread F and the structure of equatorial ionization depletions in the southern anomaly region, J. Geophys. Res., 101, 26,819-26,827, 1996.

Sastri, J. H., Post-midnight onset of spread-F at Kodaikanal during the June solstice of solar minimum, Ann. Geophys., 17, 1111-1115, 1999.

Scherliess, L. and B. G. Fejer, Storm time dependence of equatorial disturbance dynamo zonal electric fields, J. Geophys. Res., 102, 24,037-24,046, 1997.

Singh, S., D. K. Bamgboye, J. P. McClure, and F. S. Johnson, Morphology of equatorial plasma bubbles, J. Geophys. Res., 102, 20,019-20,029, 1997a

Singh, S., F. S. Johnson, and R. A. Power, Gravity wave seeding of equatorial plasma bubbles, J. Geophys. Res., 102, 8399-7410, 1997b.

Subbarao, K. S. V. and B. V. Krishna Murthy, Post-sunset F-region vertical velocity variations at magnetic equator, J. Atmos. Terr. Phys., 56, 59-65, 1994.

Weber, E. J., S. Basu, T. W. Bullett, C. Valladares, G. Bishop, K. Groves, H. Kuenzler, P. Ning, P. J. Sultan, R. E. Sheehan, and J. Araya, Equatorial plasma depletion precursor signatures and onset observed at $11^{\circ}$ south of the magnetic equator, J. Geophys. Res., 101, 26,829-26,838, 1996.

Wright, J. W., P. E. Argo, and M. L. V. Pitteway, On the radiophysics and geophysics of ionogram spread F, Radio Sci., 31, 349-366, 1996.

G. G. Bowman and I. K. Mortimer (e-mail: mortimer@physics.uq.edu.au) 